\title{
MECHANICAL BEHAVIOUR OF SMALL LOAD BEARING STRUCTURES FABRICATED BY 3D PRINTING
}

UDC: 655.3 .066 .51

Original scientific paper

https://doi.org/10.18485/aeletters.2019.4.3.2

\author{
Nikola Palić ${ }^{1}$, Vukašin Slavković ${ }^{1}$, Živana Jovanović ${ }^{1}$, Fatima Živić ${ }^{1}$, Nenad Grujović ${ }^{1}$ \\ ${ }^{1}$ University of Kragujevac, Faculty of Engineering, Sestre Janjić 6, 34000 Kragujevac, Serbia
}

\begin{abstract}
:
3D printing is state-of-the-art manufacturing technology. In addition to prototyping, the development of this technology allows the parts produced by this technology to be used as fully functional assemblies. There are different 3D printing technologies (Fused Deposition Modeling, FDM, Selective Laser Sintering, SLS, Stereolithography, SLA and others), and different materials can be used (polymer filaments, polymer and composite powders for sintering, photopolymers). This paper presents the process of design and fabrication of small load bearing structures by FDM and verification of its mechanical properties, by using three-point bending test. The printing parameters and setup of the three-point bending test were analysed from aspects of flexural strength and final bending angle that was determined experimentally and by calculation.
\end{abstract}

ARTICLE HISTORY

\section{INTRODUCTION}

3D printing enables fabrication of complex 3D structures, by depositing layer upon layer of the material, based on the virtual design of the structure. It belongs to the additive manufacturing (AM) technologies. Process involves adding material, such as liquid or powder layers. One of the main advantages of 3D printing is the ability to produce highly complex structures, from both aspects of design and used materials, based on the CAD model. Different 3D printing technologies are used today, such as Fused Deposition Modeling, FDM, Selective Laser Sintering, SLS, Stereolithography, SLA and many other specific methods are investigated and developed. 3D printing has many advantages over traditional manufacturing technologies, where customization and low cost of the small batches are among the key ones. It is used to produce prototypes during the design stages, and enables significantly shortened time from idea to the final product. Application areas are vast, from industrial elements and systems, structural elements, 3D printed electronics, medical devices, up to the recent bioprinting of living functionalized tissues and advanced multi-functional structures. It has been used to manufacture end-use products or spare parts for the systems. Some latest developments include multimaterial printing that enables fabrication of complex objects from aspect of materials. However, 3D printing is still technology in development with many details under investigation, such as the tailoring of the final part mechanical properties.

One of the mostly utilized polymer materials in FDM 3D printing is polylactic acid or polylactide (PLA). It belongs to a group of bioplastics because it is biodegradable polymer and it is heavily used in vast number of fields. It is low cost material, based on plant starch, and as such is biocompatible. It is widely used in medicine, but also in industry as the replacement of the conventional polymers. PLA advantages include ecological aspects, biocompatibility, processability and energy saving in production. It is used in production of medical scaffolds, sutures and drug delivery devices [1]. PLA is also used as decomposable packaging material, for different consumer goods (bags, food packaging, disposable tableware, etc.). On the other side, PLA has low strength, relatively slow degradation and is 
hydrophilic material, namely it attracts and holds water. Accordingly, different investigations are still realised in order to enable tailoring of the mechanical properties of the final product.

Influence of the infill properties on flexural rigidity through a parametric investigation of the frame structures manufactured by using FDM-based 3D printer is still the topic of investigations [2]. The influence of the changes in material deposition sequence on the mechanical properties is significant. Speed of printing, as well as time between deposition of two adjacent layers, affect both the tensile and flexural strength [3]. The mechanical properties of the material are influenced by the infill pattern [4]. There are different methods of mechanical testing and for structural elements, the three-point bending test is simple and efficient way to determine flexural strength of the material. Samples can be easily prepared for this test. PLA flexural strength has significant dependence on the layer thickness, during 3D printing [5]. Variation of the printing temperature and heat treatment after the printing can provide tailoring of the mechanical properties [6]. Experimental results showed that different thickness of the layers and diameter of the filament, significantly changed PLA flexural strength, whereas the speed of printing exhibited no influence $[7,8]$.

This paper presents the process of design and fabrication of small load bearing structures by FDM $3 \mathrm{D}$ printing and verification of its mechanical properties, by using PLA filament. The parameters of the sample print and the setting of the printers have been analysed. Three-point bending test was used to determine mechanical properties of the fabricated elements. Process parameters and results are analyzed.

\section{THE MAIN TITLE (CAPITAL LETTERS - CALIBRI 11 PT, BOLD) - LEFT ALIGNEMENT}

PLA specimen was produced by 3D printing from black filament ( $1.75 \mathrm{~mm}$ diameter) provided as standard material with Wanhao 3D printer. Specimen is a beam with dimensions of $120 \times 10 \times$ $2 \mathrm{~mm}$ (Fig.1 and 2). Geometry of the sample is shown in Fig. 1 and the printed PLA sample is shown in Fig.2.

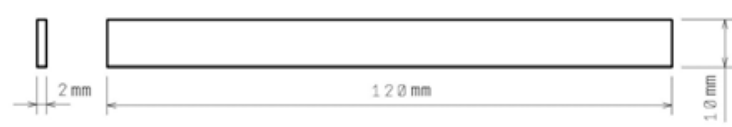

Fig.1. Geometry of the sample

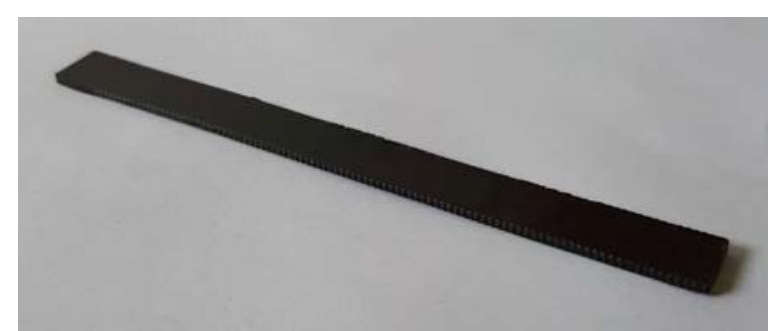

Fig.2. Printed sample by using Wanhao Duplicator I3 Plus and Wanhao PLA Black filament

All samples in this study were printed by using Wanhao Duplicator i3 Plus 3D FDM printer with 0.4 $\mathrm{mm}$ nozzle. We used standard filament made of PLA provided by the 3D printer manufacturer, in order to evaluate if this material can be used for small bearing structures. We used standard default parameters of the printer with $100 \%$ infill and layer height of $0.1 \mathrm{~mm}$, with printing speed of $50 \mathrm{~mm} / \mathrm{s}$. The estimated time for printing was $1.5 \mathrm{~h}$ and $3 \mathrm{~g}$ of material was used. All printing parameters are given in Table 1. Layer height refers to the thickness of one layer during 3D printing, and shell thickness is the thickness of the outer layers, whereas the printing head goes around the shell and then fills it with layers. Bottom-top thickness is the thickness of the initial and final printing layers, which were printed in full fill, regardless of the specified filling density parameters. Infill density refers to the filling of the object elements. Print speed refers to the speed of the printing head during the printing in a straight line. Printing temperature is the temperature of the nozzle during the printing. Sample is printed on the plate that is also heated (bed temperature), in order to avoid damages in the object due to sticking and stress distributions. Raft serves as the support on which the object is directly printed, because it enables easier removal of the final printed sample.

Table 1. 3D printing parameters at Wanhao I3 Plus printer, by using PLA material

\begin{tabular}{|c|c|}
\hline \multicolumn{2}{|c|}{ Quality } \\
\hline Layer height & $0.1 \mathrm{~mm}$ \\
\hline Shell thickness & $0.8 \mathrm{~mm}$ \\
\hline Enable retraction & Yes \\
\hline \multicolumn{2}{|c|}{ Fill } \\
\hline Bottom/Top thickness & $0.6 \mathrm{~mm}$ \\
\hline Infill density & $8 \%$ \\
\hline Print speed and temperature \\
\hline Print speed & $50 \mathrm{~mm} / \mathrm{s}$ \\
\hline Printing temperature & $210^{\circ} \mathrm{C}$ \\
\hline Bed temperature & $50^{\circ} \mathrm{C}$ \\
\hline \multicolumn{2}{|c|}{ Support } \\
\hline Support type & None \\
\hline
\end{tabular}




\begin{tabular}{|c|c|}
\hline Platform adhesion type & Raft \\
\hline \multicolumn{2}{|c|}{ Filament } \\
\hline Diameter & $1.75 \mathrm{~mm}$ \\
\hline Flow & $100 \%$ \\
\hline
\end{tabular}

Fused Deposition Modelling (FDM) is 3D printing process that uses a continuous filament of a thermoplastic material. During the printing, filament is fed through a moving, heated extruder head, where it is melted and further deposited on the pre-heated bed. Usually, printer head is computer guided during the printing process. Printer head moves in horizontal 2D plane followed with the discontinuous vertical downwards motion to start with a new layer. The speed of the extruder head may also be controlled to stop and start deposition. Wanhao Duplicator 13 is a 3D printer with a standard $1.75 \mathrm{~mm}$ filament diameter. Printer has defined set of printing parameters within its software, Wanhao-Cura (Fig.3). The stepper motor drives the filament through the $0.4 \mathrm{~mm}$ diameter nozzle. It is heated with the defined temperature to melt the filament. Preparation of the 3D model for printing is done within 3D modelling software and exported into .stl file format. This model is then imported into the 3D printer software where it is sliced into layers and positioned in relation to the printing bed. After the model is setup, printing parameters are defined: printing speed, fill density, layer height, shell thickness and whether to provide support and raft. Depending on the material nozzle temperature printing bed is selected. After the setting of all parameters $\mathrm{G}$-code is sent to the printer to execute printing of the physical model.

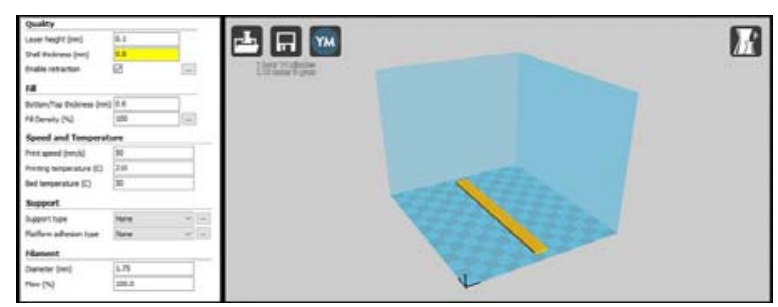

Fig.3. Working environment of the software WanhaoCura

Three-point bending test was realised at CT3 Texture Analyzer. The instrument has calibrated load cell with maximum load of $500 \mathrm{~N}$ with accuracy of $0.5 \%$. The schematic of the test is given in Fig.4.

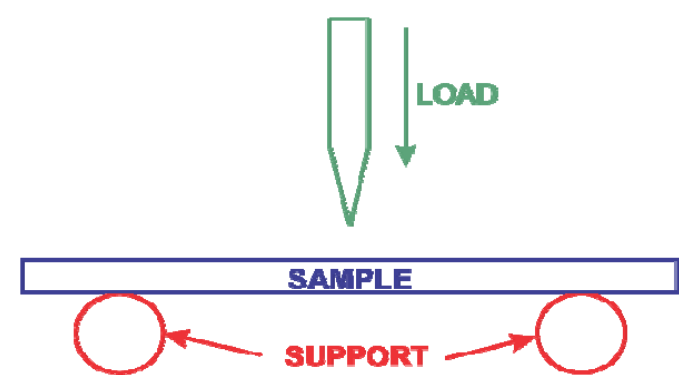

Fig.4. Scheme of the three point bending test

Test parameters can be monitored in real time within in-built software: load influence on displacement over the test time. Test data are recorded in real time, according to the frequency sampling setup at the beginning of the test. Recorded data enables calculation of stress, strain and strain rate. The sample was tested by placing it on two roller bearings (Fig.4). Speed of the indent was $0.1 \mathrm{~mm} / \mathrm{s}$ in both directions (in compression and withdrawal regimes) and the compression was stopped and indenter started to withdraw, when specified displacement is reached. Threshold displacement was $17.3 \mathrm{~mm}$, as measured from the horizontal bottom line and the test was stopped when this deflection was reached. Load vs displacement and load vs bending angle was studied.

\section{RESULTS AND DISCUSSION}

The image of the sample during the test, at the moment when the maximum bending angle is reached, is shown in Fig.5 (left image). Sample after the test is shown in Fig. 5 (right image). After the bending test is finished, the sample tends to go back to its original shape after deflection, but some deformation stayed, thus showing that this test load resulted in deformation beyond the elastic limit of the material. The final bending angle was $12^{\circ}$, after material relaxation, as shown in Fig.6.

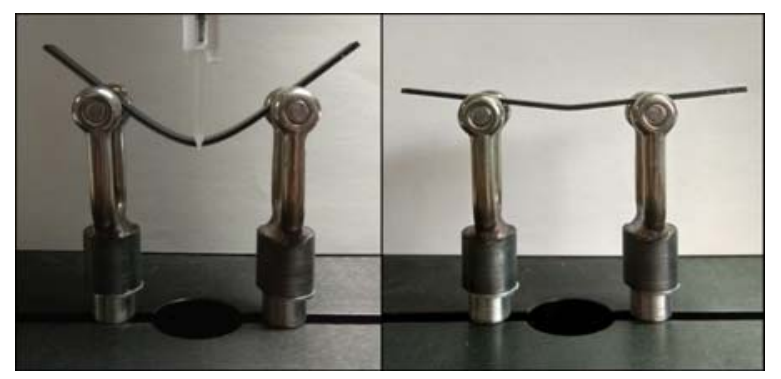

Fig.5. Maximum bent sample (left), sample after relaxation (right) 


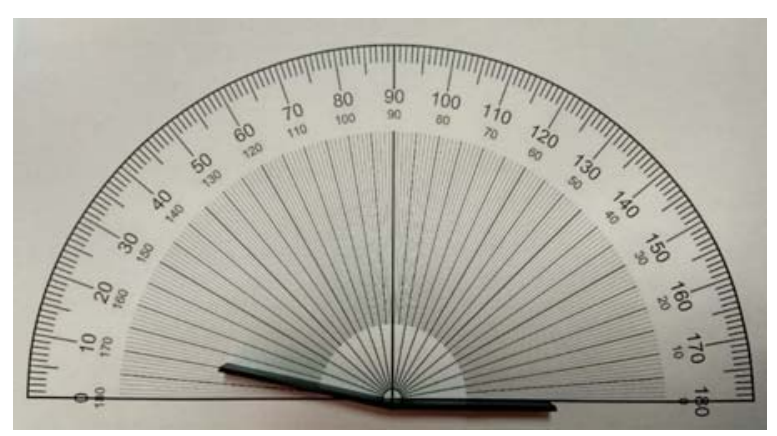

Fig.6. Bending angle after relaxation of the sample

Threshold displacement of $17.3 \mathrm{~mm}$ that was setup in the test, corresponded to the maximum compressive load of $30 \mathrm{~N}$, as shown in Fig.7. Diagram shown in Fig.7, is obtained by using the formula for bending angle calculation given in [9]. It can be seen that the bending angle of $20^{\circ}$ was exhibited at maximum load of $30 \mathrm{~N}$. After the indenter started to withdraw, thus gradually lowering the compressive load, bending of the sample still occurred until the maximum exhibited bending angle of $32^{\circ}$, under the load of $25 \mathrm{~N}$, as shown in Fig.7. After that point, the sample did not bend any more, but started to go back towards its original shape. Elastic limit of the material was reached at around $24 \mathrm{~N}$, and bending angle at that point was around $12.5^{\circ}$.

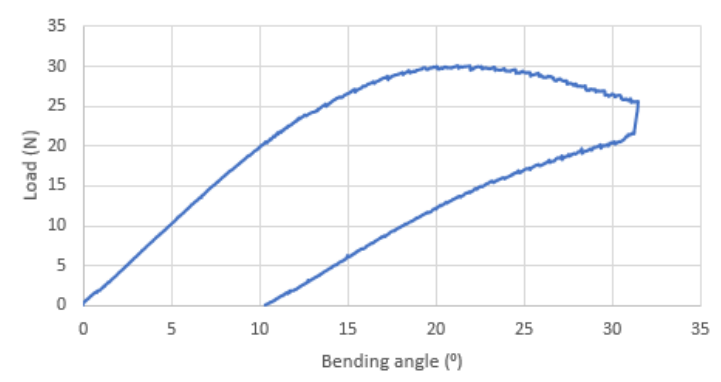

Fig.7. Changes of the bending angle as the function of the compressive load, during the three-point bending

test (calculated values according to the equation provided in [9])

According to diagram in Fig.7, the sample returned to the final bending angle of around $10.5^{\circ}$. However, experimental results showed that final bending angle was around $12^{\circ}$, when the sample was removed from the test device and material relaxed, as can be seen in Fig.6. There is the slight difference between calculated value of the final bending angle (in Fig.7) and experimentally obtained value (in Fig.6) of around $1.5^{\circ}$. Larour et al. [9] showed that different methods of bending angle calculation resulted in different values of the angle and experimental verification is needed, but there is accordance within these two values since the difference is not large.

Flexural strength of PLA sample during threepoint bending, as the function of the sample deflection, is given in Fig.8. It can be seen that the maximum flexural stress of the sample was 270 MPA, at $11.5 \mathrm{~mm}$ deflection. First micro-failures within the sample occurred at around $6.5 \mathrm{~mm}$ deflection, under flexural stress of $214 \mathrm{MPa}$, thus the flexural strength of PLA sample was $214 \mathrm{MPa}$.

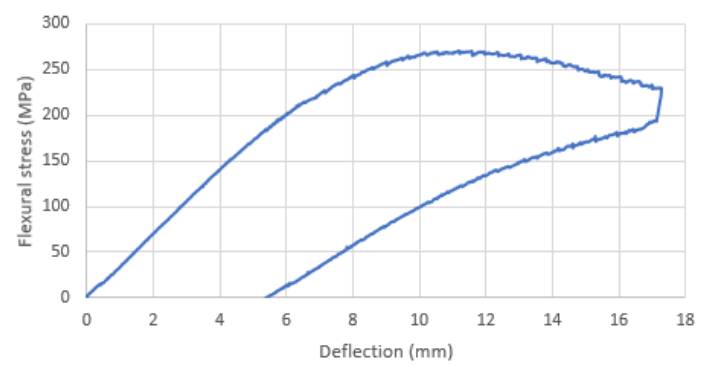

Fig.8. Flexural strength of PLA sample during threepoint bending, as the function of the sample deflection

Our results are in accordance with results of other authors [10]. Jaya Christiyan et al. [10] obtained lower values of flexural strength $(68$ $\mathrm{MPa}$ ) for the 3D printed layer of $0.2 \mathrm{~mm}$, whereas they showed significant decrease of the PLA flexural stress with increase of the layer height. Layer height during 3D printing in our work was 0.1 $\mathrm{mm}$ and higher value of the flexural strength was expected, as shown in Fig.8. In their work the samples were made of PLA material, with $40 \%$ of infill density, and during the three-point bending, indenter speed was $0.2 \mathrm{~mm} / \mathrm{s}$, whereas our samples had $100 \%$ infill, at $0.1 \mathrm{~mm} / \mathrm{s}$ speed of the indenter, thus resulting in higher flexural strength of our sample.

\section{CONCLUSION}

PLA beam was fabricated by 3D printing (FDM), with printing layer of $0.1 \mathrm{~mm}$, and it showed satisfying mechanical properties, under threepoint bend testing. Samples were printed with $100 \%$ infill and resulting flexural strength was 214 $\mathrm{MPa}$. Elastic limit of the material was reached at around $24 \mathrm{~N}$, with bending angle at that point around $12.5^{\circ}$. The final bending angle of the sample beam was $12^{\circ}$, after material relaxation and finished testing. Experimental and numerical values of the final bending angle were compared 
and they were in accordance to each other. Infill density during 3D printing significantly influence the resulting mechanical properties and further work will include variation of this parameter to determine closer dependences and provide optimal 3D printing parameters from aspect of mechanical properties of the final object.

\section{ACKNOWLEDGEMENT}

This work was supported by projects III41017, III41007, ON174028 and TR35021, Ministry of Science and Technological Development, Serbia.

\section{REFERENCES}

[1] J. Villacres, D. Nobes, C. Ayranci, Additive manufacturing of shape memory polymers: effects of print orientation and infill percentage on mechanical properties. Rapid Prototyping Journal, 24 (4), 2018: 744-751.

https://doi.org/10.1108/RPJ-03-2017-0043

[2] J. H. Porter, T. M. Cain, S. L. Fox, P. S. Harvey, Influence of infill properties on flexural rigidity of 3D-printed structural members. Virtual and Physical Prototyping, 14 (2), 2019: 148-159.

https://doi.org/10.1080/17452759.2018.1537064

[3] N. Volpato, T.T. Zanotto, Analysis of deposition sequence in tool-path optimization for low-cost material extrusion additive manufacturing. The International Journal of Advanced Manufacturing Technology, 101 (58), 2018: 1855-1863.

https://doi.org/10.1007/s00170-018-3108-1

[4] S.F. Khan, H. Zakaria, Y.L. Chong, M.A.M. Saad, K. Basaruddin, Effect of infill on tensile and flexural strength of 3D printed PLA parts, IOP Conference Series. International Conference on Advanced Manufacturing and Industry Applications (IOP Conf.), 429, 2018: 012101. https://doi.org/10.1088/1757-899X/429/1/012101

[5] A. Nugroho, R. Ardiansyah, L. Rusita, I.L. Larasati, Effect of layer thickness on flexural properties of PLA (PolyLactid Acid) by 3D printing, $6^{\text {th }}$ International Seminar of
Aerospace Science and Technology. Series: Journal of Physics: Conf. Series (IOP Conf.), 1130, 2018: 012017.

https://doi.org/10.1088/1742-6596/1130/1/012017

[6] E. Avila, J. Eo, J. Kim, N.P. Kim, Heat Treatment Effect on Mechanical Properties of 3D Printed Polymers. $2^{\text {nd }}$ International Conference on Composite Material, Polymer Science and Engineering (CMPSE2018), 264, 2019: 02001.

https://doi.org/10.1051/matecconf/201926402001

[7] K.G. Jaya Christiyana, U. Chandrasekharb, N. Rajesh Mathivanan, K. Venkateswarlu, Influence of manufacturing parameters on the strength of PLA parts using Layered Manufacturing technique: A statistical approach, IOP Conf. Series: Materials Science and Engineering, 310, 2018: 012134.

https://doi.org/10.1088/1757-899X/310/1/012134

[8] J. Villacres, D. Nobes, C. Ayranci, Additive manufacturing of shape memory polymers: effects of print orientation and infill percentage on mechanical properties. Rapid Prototyping Journal, 24 (4), 2018: 744-751.

https://doi.org/10.1108/RPJ-03-2017-0043

[9] P. Larour, B. Hackl, F. Leomann, K. Benedyk, Bending Angle Calculation In the Instrumented Three-Point Bending Test. IDDRG Conference 2012, "Lightweighting: Possibilities \& Challenges", Mumbai, India, Vol.C/25/12, 2012.

[10] K. G. Jaya Christiyan, U. Chandrasekhar, K. Venkateswarlu, Flexural Properties of PLA Components Under Various Test Condition Manufactured by 3D Printer. Journal of The Institution of Engineers (India): Series C, 99, (3), 2018: 363-367.

https://doi.org/10.1007/s40032-016-0344-8

An expanded abstract of this research was presented at " 9 th International Scientific Conference IRMES 2019", September 5-7, 2019, Kragujevac, Serbia. 